\section{Study of Photoneutron Production for the 18 MV Photon Beam of the Siemens Medical linac by Monte Carlo Simulation}

\author{
Dowlatabadi H. ${ }^{1 * \bullet}$, Mowlavi A. A. ${ }^{2,3}$, Ghorbani M. ${ }^{4}$, Mo- \\ hammadi S. ${ }^{1}$, Knaup C. ${ }^{5}$
}

\begin{abstract}
Background: Considering the importance of photoneutron production in linear accelerators, it is necessary to describe and measure the photoneutrons produced around modern linear accelerators.

Objective: The aim of the present research is to study photoneutron production for the $18 \mathrm{MV}$ photon beam of a Siemens Primus Plus medical linear accelerator.

Material and Methods: This study is an experimental study. The main components of the head of Siemens Primus Plus linac were simulated using MCNPX 2.7.0 code. The contribution of different components of the linac in photoneutron production, neutron source strength, neutron source strength and photon and electron spectra were calculated for the flattening filter and flattening filter free cases for the $18 \mathrm{MV}$ photon beam, and was scored for three fields of $5 \times 5 \mathrm{~cm}^{2}, 10 \times 10 \mathrm{~cm}^{2}$ and $20 \times 20 \mathrm{~cm}^{2}$ in size.
\end{abstract}

Results: The results show that the primary collimator has the largest contribution to production of neutrons. Moreover, the photon fluence for the flattening filter free case is 8.62, 6.51 and 4.62 times higher than the flattening filter case for the three fields, respectively. The electron fluences for the flattening filter free case are 4.62, 2.93 and 2.79 times higher than with flattening filter case for the three fields under study, respectively. In addition to these cases, by increasing the field size, the contribution of neutron production related to the jaws is reduced, so that when the field size increases from $5 \times 5 \mathrm{~cm}^{2}$ to $20 \times 20 \mathrm{~cm}^{2}$, a $17.93 \%$ decrease in photoneutron production was observed.

Conclusion: In all of the accelerators, the neutron strength also increases with increasing energy. The calculated neutron strength was equal to $0.83 \times 10^{12}$ neutron Gy ${ }^{-1}$ at the isocenter.

Citation: Dowlatabadi H, Mowlavi AA, Ghorbani M, Mohammadi S, Knaup C. Study of Photoneutron Production for the 18 MV Photon Beam of the Siemens Medical linac by Monte Carlo Simulation. J Biomed Phys Eng. 2020;10(6):679-690. doi: 10.31661/jbpe.v0i0.939.

\section{Keywords}

Neutron Contamination; Particle Accelerators; 18 MV Photon Beam; Monte Carlo Method; Electrons; Neutron Source Strength; Proton Spectrum

\section{Introduction}

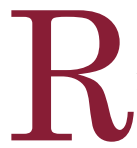
adiotherapy is currently an essential component in the management of cancer patients, and radiotherapy using electron linear accelerators (linac) has an increasing role in treatment of cancerous tumors. On the other hand, medical linacs which operate above 10 MV for high energy photon therapy inevitably produce neutron contamination. Neutrons are produced through $(\gamma, \mathrm{n})$ photonuclear reaction
${ }^{1} \mathrm{PhD}$, Physics Department, School of Sci-

ences, Payame Noor University of Mashhad,

Mashhad, Iran

${ }^{2}$ PhD, Physics Depart-

ment, School of Sci-

ences, Hakim Sabzevari

University, Sabzevar, Iran

${ }^{3} \mathrm{PhD}$, International Cen-

tre for Theoretical Phys-

ics, Associate Federation

Scheme, Medical Phys-

ics Field, Trieste, Italy

${ }^{4} \mathrm{PhD}$, Biomedical En-

gineering and Medical

Physics Department,

Faculty of Medicine,

Shahid Beheshti Univer-

sity of Medical Sciences,

Tehran, Iran

${ }^{5} \mathrm{PhD}$, Comprehensive

Cancer Centers of

Nevada, Las Vegas,

Nevada, USA

*Corresponding author:

H. Dowlatabadi

Physics Department,

School of Sciences,

Payame Noor University

of Mashhad, Mashhad, Iran

E-mail: h_d_ph@yahoo. com

Received: 13 May 2018 Accepted: 5 June 2018 
in the components of the head of linacs [1-3].

The energy threshold for a photonuclear reaction depends on the atomic number of material $(Z)$, and by increasing the value of $Z$ the energy threshold decreases [4-5]. Neutrons produced in the head of a linac have an energy range of 1-2 MeV for 10-18 MV X-ray beams, and are more hazardous than photons in causing radiation-induced late effects [6]. Neutrons have a radiation quality factor higher than radiation quality factor for photons. This quantity shows that the biological effects of neutrons are higher than those of photons. The quality factor for neutrons ranges from 1 to 20 [7].

Neutrons are sources of biological damage owing to their high radiation quality factor. The neutron dose received by the body leads to an increased risk of induction of secondary cancers outside the treatment region. There are many studies on the induction of secondary cancers following radiotherapy. For example Nyandoto et al., [8] stated in their study that during a follow-up consisting 4020 personyears, 27 patients developed secondary cancer. Kleinerman et al., [9] mentioned in their study that among the 49,828 women treated with radiation, 3750 cases survived 30 or more years and a two-fold risk of cancers of irradiated organs was seen. Anil et al., [10] stated in their study that cervical cancer patients treated with radiotherapy are at increased risk of second cancers at sites in close proximity to the cervix [11].

Owing to the importance of the neutrons produced, it is necessary that neutron doses be considered in radiotherapy treatment. Measuring the neutron dose in the patient's body using neutron detectors is not easy. Therefore, the use of an accurate computational code is a useful tool to achieve this goal. Monte Carlo (MC) methods have been widely used in medical radiation physics, especially in radiation therapy physics. One of the most popular MC software packages that have been used in a large number of applications is the Monte Car- lo N-Particle (MCNP) transport code package and the present study was performed using this package.

Several studies have been performed on the investigation of the effect of neutron doses to patients undergoing radiation therapy, and on neutron contamination dosimetry around high-energy linacs for different types and energies of linacs. Zabihinpoor et al., [12] in their study used Neptune accelerator to investigate neutrons. Their results indicate that when the distance from the isocenter decreases, production of photoneutrons will increase. And as the radiation field increases, neutron production increases.

Martinez et al., [13] calculated the neutron dose for 15 and $18 \mathrm{MV}$ photon beam of Varian $2100 \mathrm{C} / \mathrm{D}$ and $15 \mathrm{MV}$ photon beam of Elekta linac. Their results show the amount of neutrons produced by $15 \mathrm{MV}$ linacs is smaller than that found for the $18 \mathrm{MV}$ linacs. Ma et al., [14] used MCNPX code for simulations. Simulations were performed for various field sizes which are most often used in radiation therapy.

In this study, a detailed geometry of $18 \mathrm{MV}$ Siemens Primus Plus linear accelerator head was simulated using the Monte Carlo method. In this accelerator, the flattening filter which is made of stainless steel is more complex, and different from that which is used for the $6 \mathrm{MV}$. Due to the complex structure of the flattening filter, and due to different geometries of the jaws and various components of the Siemens Primus Plus accelerator, neutron production in this accelerator is different from other accelerators.

The aim of the present research is to study photoneutron production for the $18 \mathrm{MV}$ photon beam and the effect of flattening filter on electron and photon spectrum for Siemens Primus Plus medical linear accelerator using MCNPX code. Several studies were performed on the neutron contamination around various linacs. These studies were performed with different computational codes [15-16]. 
Study of Photoneutron Production for the 18 MV Photon Beam

\section{Materials and Methods}

This study is an experimental study. Different components of the Siemens Primus Plus accelerator, with $18 \mathrm{MV}$ photon beam, were considered for studying the production of neutrons in various fields. In the present study, contribution of the linac head components to photoneutron production, neutron source strength, and the effects of flattening filter on electron and photon spectra were calculated. The reason for calculation of the effect of flattening filter on photon and electron energy spectra is that neutron production is based on the interaction of electrons and photons with linac's head components and patient's body. When the photon and electron energy spectra change, the neutron production also changes. It is known that flattening filter absorbs photons and electrons and by removing flattening filter, photon and electron energy spectra are increased. For this reason, the photon and electron energy spectra were obtained on the phantom surface to determine that there are neutrons on the phantom surface. According to the photon and electron energy spectra, it can be seen that in high energy beams a number of peaks are observed (though negligible). These small photon and electron components can produce neutron which have destructive biological effects.

\section{Simulation of Siemens Primus Plus linac}

As the first step of the present study, a Siemens Primus Plus medical linac was simulated using MCNPX software (version 2.7.0). Validation of this model was performed in a previous study by Dowlatabadi et al, [17]. The Siemens Primus Plus medical linac has two treatment modes: photon and electron. This linac works with two photon energies (6 and $18 \mathrm{MV}$ ) and five electron energies (6, $8,10,12,15$ and $18 \mathrm{MeV})$ at the treatment center of this study. The geometric characteristics of the accelerator (composition and density) were provided by the linac's manu- facturer. The comparisons of dose profile and PDD (Percent Depth Dose) values obtained by MC simulations and measurements were performed by calculation of gamma function. For dose profiles and PDD data, the agreement was obtained between two sets of the data for $5 \times 5 \mathrm{~cm}^{2}, 10 \times 10 \mathrm{~cm}^{2}$ and $20 \times 20 \mathrm{~cm}^{2}$ field sizes. Dose profile in more than $90 \%$ of the points of the simulations had gamma values less than unity, and for PDD, nearly all data of the points of the simulations had gamma values less than unity. Gamma functions between zero and unity are considered as pass or agreement, while gamma values higher than unity are considered as fail or disagreement.

The geometry of the Siemens Primus Plus medical linac's head is illustrated in Figure 1 and the material compositions of the simulated
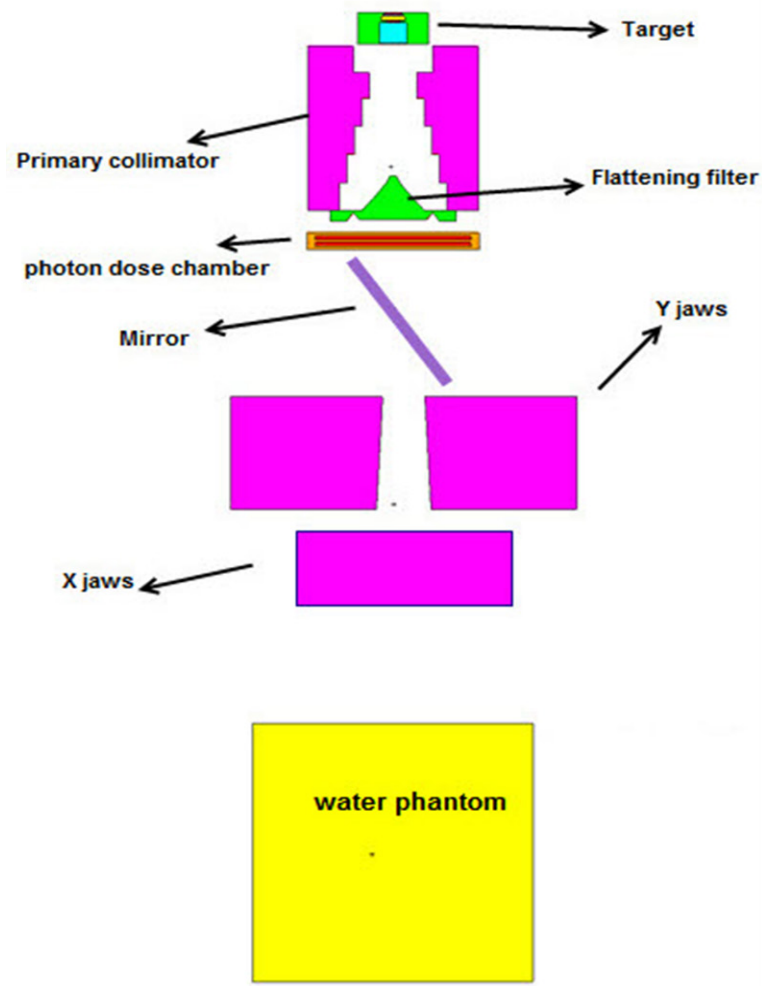

Figure 1: Schematic representation of the Siemens Primus Plus linac's head geometry in the photon mode ( $Y-Z$ view). The water phantom was defined as a cube with dimensions of $50 \mathrm{~cm} \times 50 \mathrm{~cm} \times 50 \mathrm{~cm}$. 
components of the linac's head are tabulated in Table 1. The head of the linac contains the following components: target, absorber, flattening filter, primary collimator, photon monitoring chamber, mirror and jaws. The target has a cylindrical shape with a height of about 1.5 $\mathrm{cm}$ and a diameter of approximately $3 \mathrm{~cm}$. The flattening filter is made of stainless steel and is located at a distance of $4 \mathrm{~cm}$ from the target. The primary collimator is made of tungsten and is located under the target at distance of $1.54 \mathrm{~cm}$ from the target, having a height of about $6.52 \mathrm{~cm}$. In addition to the head components, a water phantom was simulated as well. A cubic water phantom with dimensions of $50 \mathrm{~cm} \times 50 \mathrm{~cm} \times 50 \mathrm{~cm}$ was simulated, the surface of which was $100 \mathrm{~cm}$ away from the target.

In order to reduce the time of calculations, energy cut-off was considered and other methods of variance reduction were not used. The energy cut-off for photons and electrons was set to $7 \mathrm{KeV}$. The importance of the particle in each volume is represented by the magnitude of importance (IMP). If a cell importance is set to zero for a particle, the importance for tracking that particle in that cell will be set to zero.

The cell importance for both electrons and photons was set as 100 for cells that are con-

Table 1: Materials of the various components in the $18 \mathrm{MV}$ photon beam energy of the Siemens Primus Plus linac. The characteristics of the accelerator geometry were supplied by the manufacturer, Siemens.

\begin{tabular}{cc} 
Component & Material \\
\hline Jaws & Tungsten \\
\hline Mirror & Silicon oxide $\left(\mathrm{SiO}_{2}\right)$ \\
\hline Target & Gold \\
\hline Absorber & Aluminum \\
\hline Flattening filter & Stainless steel alloy $(\mathrm{SST}-303)$ \\
\hline Primary collimator & Tungsten \\
\hline Photon dose chamber & Aluminum oxide $\left(\mathrm{Al}_{2} \mathrm{O}_{3}\right)$
\end{tabular}

sidered in the calculations. The MC simulations in the present study were run on a computer with an Intel ${ }^{(\mathrm{R})}$ core $^{\mathrm{TM}} \mathrm{i} 7$ processor, 4.00 $\mathrm{GHz}$, featuring a 64-bit operating system.

Benchmark comparison was performed by comparing the simulated PDD data for the 5 $\times 5 \mathrm{~cm}^{2}, 10 \times 10 \mathrm{~cm}^{2}$ and $20 \times 20 \mathrm{~cm}^{2}$ field sizes with the corresponding measured data. In addition, the dose profile data for different depths and energies for the $5 \times 5 \mathrm{~cm}^{2}, 10 \times$ $10 \mathrm{~cm}^{2}$ and $20 \times 20 \mathrm{~cm}^{2}$ field sizes were compared with the corresponding measured data, as it was mentioned in the previous study by Dowlatabadi et al, [17].

The dose profiles showed good agreement with the measurement data for all depths. The configuration of the simulated dose profiles matched well with the measurement at the central areas of the dose profiles.

Contribution of the linac head components to photoneutron production

All the simulations in this section were performed for a $10 \times 10 \mathrm{~cm}^{2}$ field size. The main components that have contribution to production of photoneutrons in a linac's head are those with high atomic numbers, including the target, primary collimator, flattening filter and jaws. Lead and tungsten are the main elements that produce photoneutrons in different medical linacs, because their cross-sections for $(\gamma$, n) reaction are high. The energy threshold of photoneutron production for tungsten and lead are 7.19 MeV and 6.74 MeV, respectively [7]. In this section of the study, calculation method of contribution of the linac head components to photoneutron production is expressed. In order to obtain the contribution of each component in production of neutrons, calculations were performed using three methods which are explained in the following and the results were compared together. After comparing, the best method with the least discrepancies was selected.

The first method: in this step the MPN card was considered to be zero for all the compo- 
nents except for the desired component (when MPN command is considered to be zero, production of neutrons in that component is not considered). Subsequently, after running the programs, the obtained values were divided to the total values (the total number of produced photoneutrons). In other words, the ratio of the number of neutrons produced from these components to the total number of neutrons produced from all parts of the head of the accelerator was then calculated. By this way, contributions of the linac's head components in photoneutron production were obtained.

The second method: in this step each component, such as the jaws, flattening filter and primary collimator, were selected as a volume. The number of neutrons entering into the surfaces corresponding to the desired volumes was then subtracted from the number of neutrons exiting from the surfaces. F1 tally was used to calculate the number of particles entering each surface. However, since F1 tally gives the number of particles passing through the surface regardless of their direction, a " $\mathrm{Cn}$ " card was used to segment the exit directions of the particles from the desired level compared to the normal vector. With segmentation of the particle output angle in the range of $0^{\circ}$ to $90^{\circ}$, and $90^{\circ}$ to $180^{\circ}$ compared to the normal vector, and by subtraction of the input and output values, the number of neutrons produced in the desired volume was obtained.

The third method: in this step of study to run the program, "primt table 140" card was used to account for neutron production. In the MCNP code, the primt table 140 card is used to obtain photoatomic activity of each nuclide in each cell.

Energy cut-off for electrons and photons was set to $7 \mathrm{keV}$. Additionally, to calculate the neutron contamination, the energy cut-off for photons and electrons was set to $7 \mathrm{MeV}$. In the MCNP code, PHYS, MPN and MX card are used to obtain neutron quantities. For this reason, in this study in order to account for neutron production from $(\gamma, n)$ interactions,
"PHYS", "MPN", and "MX" cards were used. The PHYS command is used to specify the energy cut-off and the physics treatments. Additionally, in this program, "MT" card was used to calculate thermal neutron scattering. In these simulations, the type A uncertainties were below $1.9 \%$.

\section{Effect of field size on photoneu- trons production}

Owing to the relatively large contribution of jaws to neutron production, it is necessary to question to what degree the size of the radiation field affects the production of neutrons. To answer this question, the amount of neutrons produced at the isocenter by interaction of photons with the jaws was calculated in separate programs for the $5 \times 5 \mathrm{~cm}^{2}, 10 \times 10$ $\mathrm{cm}^{2}$ and $20 \times 20 \mathrm{~cm}^{2}$ field sizes. In this step, the PHYS, MPN and MX card were used.

\section{Neutron source strength}

One of the quantities that used to describe the production of neutrons in a linac is neutron source strength (neutron yield) which is represented by the $Q$ quantity. The neutron source strength represents the number of neutrons produced per 1 Gy photon absorbed dose. To calculate this value, according to the method proposed by McGinley and Landry [18], a spherical surface with radius of $100 \mathrm{~cm}$ was considered while the center of the spherical surface was positioned on the target. The study by McGinley and Landry described a method to determine the total neutron fluence $\left(\mathrm{n} / \mathrm{cm}^{2}\right)$ per unit X-ray dose at isocenter produced by several different linacs. The total neutron fluence is given by the sum of the direct, scattered, and thermal neutron fluences. F1 and F6 scoring tallies were used to calculate the photoneutrons fluence and photon air kerma at the isocenter, respectively.

The neutron fluence from the accelerator at the isocenter in terms of Gy can be calculated from the following formula (according to IAEA SRS-47 report) (International Atomic 
Energy Agency Safety Reports Series) [19, 20]:

$$
\varphi\left(n . c m^{-2} G y^{-1}\right)=\frac{a Q}{4 \pi d^{2}}+\frac{5.4 a Q}{S}+\frac{1.26 Q}{S}
$$

In this formula $a$ is the transmission factor for the head shielding ( 0.85 for tungsten and 1 for lead, we considered a $=0.85$ for simplicity), $d$ is the distance from the target to the fluence-measuring point and $Q$ is the neutron source strength. In this study, after running the program, contribution of the scattered and thermal neutron fluences was negligible, for this reason this part of fluences was neglected. According to the method proposed by McGinley and Landry, with using relationship (1) it is feasible to calculate the neutron source strength value for the $18 \mathrm{MV}$ photon beam of the Siemens primus plus medical linac and the $Q$ values for different linac models with different energies were reported.

Effect of flattening filter on electron and photon spectra

The reason for calculating the photon and electron spectrum in this section is to evaluate the influence of these spectra on the production of neutrons. By removing the flattening filter, the photon and electron spectra are changed. In this step of the study to run the program, photon and electron spectra were calculated at distance of $100 \mathrm{~cm}$ from the source on the surface of the phantom for two cases, with flattening filter and without flattening filter for the $18 \mathrm{MV}$ photon beam. In this step of the study photon and electron spectra were scored for the $5 \times 5 \mathrm{~cm}^{2}, 10 \times 10 \mathrm{~cm}^{2}$ and $20 \times 20 \mathrm{~cm}^{2}$ field sizes.

To calculate the electron spectrum, cells were in the form of cylinders with thicknesses of $0.001 \mathrm{~mm}$ were selected on the surface of the phantom. In this step, the F4 tally and E4 card were used to calculate the numbers of electrons crossing the cell.

\section{Results}

The contribution of different components of the linac to the production of neutrons for the $10 \times 10 \mathrm{~cm}^{2}$ field is shown in Figure 2 and it is compared with other Siemens accelerators. In order to obtain the contribution of each component in production of neutrons, calculations were performed using the three mentioned methods and were compared together.

After the comparison, the best method with the least discrepancy was selected. In these calculations, the maximum statistical type an uncertainty in the MC calculations for the first, second and third methods were 1.1\%, 1.9\% and $0.64 \%$, respectively. Among the three suggested methods, according to the results obtained in the output results of the program, the lowest statistical uncertainty of third method indicates the fine accuracy of this method. According to the data in Figure 2, the primary collimator has the largest contribution to production of neutrons, such that $59 \%$ of the total number of neutrons is produced by interaction of photons in the primary collimator. This result may be reasonable, because the primary collimator is made of tungsten. Tungsten has the lowest threshold energy in comparison to the other substances in the head of the accelerator for photonuclear reaction.

It can be seen from these results that, after the primary collimator, the largest contribu-

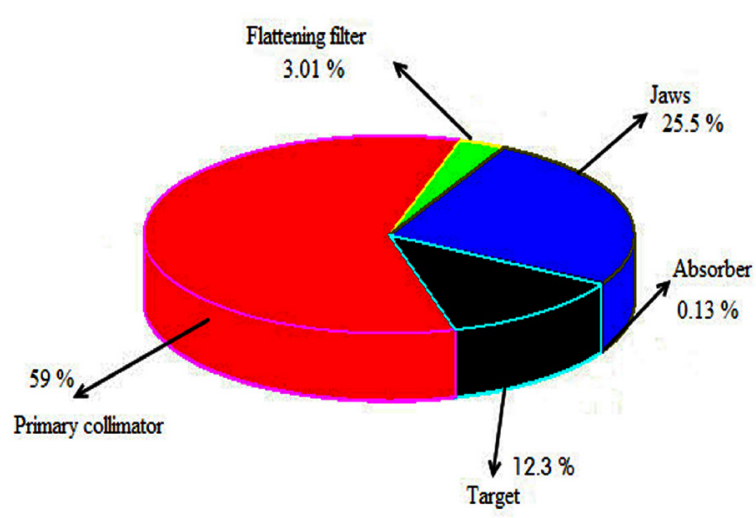

Figure 2: Neutron contribution (\%) for various components of the Siemens Primus Plus accelerator (18 MV photon beam). 
tion to production of photoneutrons is related to the jaws with a value of $25.5 \%$. In most medical linear accelerators, the jaws are made of tungsten. Therefore, they are important components in production of neutrons and become the main sources of neutron production.

The high energy photons pass through the flattening filter, and we expect that the flattening filter has a greater contribution to producing neutrons but the results are opposite and contribution to producing neutrons is negligible. According to Figure 2, the contribution of the flattening filter to production of neutrons is $3.01 \%$. The reason for this low contribution is the dominance of lighter elements, such as carbon, manganese, silicon, chromium, nickel, and iron in the flattening filter, that have higher energy thresholds for photonuclear interaction. The primary collimator and jaws are significant sources of neutrons.

The contribution of the jaws to production of neutrons for various radiation field sizes is listed in Table 2. It can be seen from the table that, by increasing the radiation field size, the con- tribution of the jaws to production of neutrons decreases. The reason for this trend is that, by increasing the field size, the opening angle of the jaws increases. Therefore, less material is placed in the path of the photon beam. For this reason, the number of photon interactions with tungsten in the jaws is reduced and the number of neutrons produced becomes lower. Mesbahi et al., [15], using a simulation of Varian accelerator (18 MV photon beam), evaluated the effect of field size on production of neutrons. Their study showed that with increasing field size, the neutron fluence decreases.

\section{Discussion}

The results of this study on the contribution of linac components are compared with the other models of Siemens linac in Table 3 $[7,16]$. MC calculations for the component contributions to photoneutrons for Varian 2100C/2300C medical linear accelerator are listed in Table 4 [19]. According to Tables 3 and 4, the differences between the data of these accelerators are due to different mate-

Table 2: Percentage value (\%) of neutron production from the jaws of the Siemens Primus Plus medical linac for different field sizes.

\begin{tabular}{lccc} 
& $5 \times 5 \mathbf{c m}^{2}$ & $10 \times 10 \mathbf{c m}^{2}$ & $\mathbf{2 0} \times \mathbf{2 0} \mathbf{c m}^{2}$ \\
\hline Neutron production in jaws (\%) & $26.82 \% \pm 0.05$ & $25.51 \% \pm 0.02$ & $22.01 \% \pm 0.03$
\end{tabular}

Table 3: Percentage (\%) contribution of each component to the production of photoneutrons for the Siemens Primus Plus medical linac (15 MV and $18 \mathrm{MV}$ photon beams).

\begin{tabular}{cccc} 
Component & $\begin{array}{c}\text { Siemens Primus } \\
(\mathbf{1 5 ~ M V ~ [ 7 ] ) ~ ( \% ) ~}\end{array}$ & $\begin{array}{c}\text { Siemens Primus } \\
\text { (15 MV [16]) (\%) }\end{array}$ & $\begin{array}{c}\text { Siemens Primus Plus (18 MV) (This study) } \\
\text { (\%) }\end{array}$ \\
\hline Target & $10.08(\mathrm{Au})$ & $12.43(\mathrm{Au})$ & $12.33(\mathrm{Au})$ \\
\hline Primary collimator & $54.85(\mathrm{~W})$ & $52.29(\mathrm{~W})$ & $59.01(\mathrm{~W})$ \\
\hline Flattening filter & $1.74(\mathrm{SST})$ & $0.41(\mathrm{SST})$ & $3.01(\mathrm{SST})$ \\
\hline Jaws & $26.72(\mathrm{~W})$ & $21.14(\mathrm{~W})$ & $25.51(\mathrm{~W})$ \\
\hline Absorber & $0.01(\mathrm{Al})$ & $0.00(\mathrm{Al})$ & $0.13(\mathrm{Al})$ \\
\hline Others & 6.6 & 13.73 & 0.01 \\
\hline Total & 100 & 100 & 100
\end{tabular}


Table 4: Percentage (\%) contribution of each component to the production of photoneutrons for the Varian $2100 \mathrm{C} / 2300 \mathrm{C}$ linac.

\begin{tabular}{|c|c|c|c|c|c|}
\hline Component & $\begin{array}{c}\text { Varian } \\
(10 \mathrm{MV}[3,19]) \\
(\%)\end{array}$ & $\begin{array}{c}\text { Varian } \\
(15 \mathrm{MV}[3,19]) \\
(\%)\end{array}$ & $\begin{array}{c}\text { Varian } \\
\text { (20 MV }[3,19]) \\
(\%)\end{array}$ & $\begin{array}{c}\text { Varian } \\
(18 \mathrm{MV}[3,19]) \\
(\%)\end{array}$ & $\begin{array}{c}\text { Varian } \\
\text { (15 MV [19]) } \\
(\%)\end{array}$ \\
\hline Target & $0.013(\mathrm{Cu})$ & $9.2(\mathrm{~W}, \mathrm{Cu})$ & $17.1(\mathrm{~W}, \mathrm{Cu})$ & $15.5(\mathrm{~W}, \mathrm{Cu})$ & $9.0(\mathrm{~W}, \mathrm{Cu})$ \\
\hline Primary & $44.2(\mathrm{~W})$ & $38(W)$ & $35.5(\mathrm{~W})$ & $39.9(W)$ & $38.0(\mathrm{~W})$ \\
\hline collimator & $0.034(\mathrm{Cu})$ & $22.4(\mathrm{~W})$ & 10.3 (Fe,Ta) & $9(\mathrm{Fe}, \mathrm{Ta})$ & $24.3(\mathrm{~W})$ \\
\hline Flattening filter & $54.7(\mathrm{~W})$ & $29.1(\mathrm{~W})$ & $36(\mathrm{~W})$ & $34.2(\mathrm{~W})$ & $22(W)$ \\
\hline Jaws & 1 & 1.3 & 1.1 & 1.4 & 5.2 \\
\hline Others & 0.0 & 0.08 & 0.0 & 0.0 & 1.5 \\
\hline Total & 100 & 100 & 100 & 100 & 100 \\
\hline
\end{tabular}

rial compositions in the heads of these linacs. By comparing Siemens Primus Plus (18 MV photon beam) and Varian 2100C/2300C linac (18 MV photon beam), it is seen that the percentage contribution of each component to the production of photoneutrons for both linac is quite different. Differences between the data of these accelerators with $18 \mathrm{MV}$ photon beams are due to different material compositions in the heads of these linacs. In the Varian accelerator, the flattening filter is made of tungsten but in the Siemens accelerator the flattening filter is made of stainless steel.

The reason for the differences in the neutron contribution of the target is the low threshold energy for tungsten compared to copper and aluminum. Additionally, for the jaws and primary collimator, the reason for the differences in the contribution of neutrons is the low threshold energy of lead compared to tungsten. The flattening filter in the Siemens Primus Plus, owing to the low atomic number and high threshold energy, has a smaller contribution to the production of neutrons compared to the production of neutrons from other accelerators.

The results of different studies on neutron source strengths of various linacs are listed in Table 5. It can be seen from the table that, with increasing photon energy, the neutron source strength increases. In other words, the neutron strength for different linacs depends on the photon energy and the linacs' head structures and the linacs' model. For the Varian linac, the neutron strength increases significantly from $10 \mathrm{MeV}$ to $18 \mathrm{MeV}$, and it reaches from 0.059 $\times 10^{12}$ neutron per Gy to $2.9 \times 10^{12}$ at the isocenter. For the Siemens primus plus linac the neutron strength increases significantly from $15 \mathrm{MeV}$ to $20 \mathrm{MeV}$, and it reaches from $0.2 \times$ $10^{12}$ neutron per Gy to $0.92 \times 10^{12}$ at the isocenter.

The photon and electron spectra at distance of $100 \mathrm{~cm}$ from the source on the surface of the phantom were scored for the $5 \times 5 \mathrm{~cm}^{2}, 10$ $\times 10 \mathrm{~cm}^{2}$ and $20 \times 20 \mathrm{~cm}^{2}$ field sizes. The photon energy spectrum for the two cases, with flattening filter and without flattening filter in $18 \mathrm{MV}$ photon beam, on the phantom surface for the $5 \times 5 \mathrm{~cm}^{2}, 10 \times 10 \mathrm{~cm}^{2}$ and $20 \times 20 \mathrm{~cm}^{2}$ field sizes are shown in Figure 3. In this diagram, the photon fluence is higher for the nonfilter case for all the three field sizes. Based on this diagram, the photon fluence per incident electron for the non-filter case are 4.62, 2.93 and 2.79 times compared to the with flattening filter case for the $5 \times 5 \mathrm{~cm}^{2}, 10 \times 10 \mathrm{~cm}^{2}$ and $20 \times 20 \mathrm{~cm}^{2}$ field sizes, respectively.

It can be seen that, as the field size decreases, the photon fluence increases for the non-filter case in comparison to the flattening filter case. When the field size increases, more photons 
Table 5: Comparison of the neutron source strength (neutron $\mathrm{Gy}^{-1}$ ) of various medical linacs.

\begin{tabular}{ccccc} 
Manufacturer & Model & Energy $(\mathbf{M V})$ & $\mathbf{Q}\left(\right.$ neutron Gy $\left.\mathbf{~}^{-1}\right)$ & Reference \\
\hline Siemens & Primus plus & 18 & $0.83 \times 10^{12}$ & This study \\
\hline Siemens & Primus & 20 & $0.92 \times 10^{12}$ & Naseri and Mesbahi [3] \\
\hline Siemens & Primus & 15 & $0.17 \times 10^{12}$ & Pena et al [16] \\
\hline Siemens & Primus & 20 & $0.92 \times 10^{12}$ & McCall [21,22] \\
\hline Siemens & Primus & 15 & $0.20 \times 10^{12}$ & Lin et al [23] \\
\hline Siemens & Primus & 15 & $0.136 \times 10^{12}$ & Becker et al [24] \\
\hline Siemens & Primus & 18 & $0.88 \times 10^{12}$ & Followill et al [25] \\
\hline Siemens & Primus & 15 & $0.2 \times 10^{12}$ & Followill et al [25] \\
\hline Siemens & Primus & 15 & $0.2 \times 10^{12}$ & Mohammadi et al [26] \\
\hline Varian & $1800 \mathrm{C}$ & 18 & $2.27 \times 10^{12}$ & McGinley and Landry [18] \\
\hline Varian & $1800 \mathrm{C}$ & 15 & $1.23 \times 10^{12}$ & McGinley and Landry [18] \\
\hline Varian & $1800 \mathrm{C}$ & 10 & $0.06 \times 10^{12}$ & McGinley and Landry [18] \\
\hline Varian & $1800 \mathrm{C}$ & 18 & $2.9 \times 10^{12}$ & McCall [22] \\
\hline Varian & $20 \mathrm{C}$ & 15 & $0.93 \times 10^{12}$ & McCall [22] \\
\hline Varian & $18 \mathrm{C}$ & 10 & $0.059 \times 10^{12}$ & McCall [22]
\end{tabular}

are crossed within the flattening filter and the ratio of the photon fluence for the non- flattening filter case compared with the flattening filter case is decreased. From Figure 3, it can be seen that the ratio of the photon fluence for the non-filter case in comparison to the flattening filter case decreases from 8.62 to 4.62 with the increase in the field size from $5 \times 5 \mathrm{~cm}^{2}$ to $20 \times 20 \mathrm{~cm}^{2}$, respectively.

The electron energy spectrum on the surface of the phantom for the $5 \times 5 \mathrm{~cm}^{2}, 10 \times 10 \mathrm{~cm}^{2}$ and $20 \times 20 \mathrm{~cm}^{2}$ field sizes is shown in Figure 4 (for the non-flattening filter case and with flattening filter case). The electron fluences for the non-flattening filter case are 4.62, 2.93, and 2.79 times compared to the flattening filter case for the $5 \times 5 \mathrm{~cm}^{2}, 10 \times 10 \mathrm{~cm}^{2}$ and $20 \times 20 \mathrm{~cm}^{2}$ field sizes, respectively. It can be mentioned that, with decreasing field size, the electron fluence increases for the non-flattening filter case analogously to the flattening filter case. These findings are in close agreement with those reported by Mesbahi et al., [15] and Kry et al, [21].
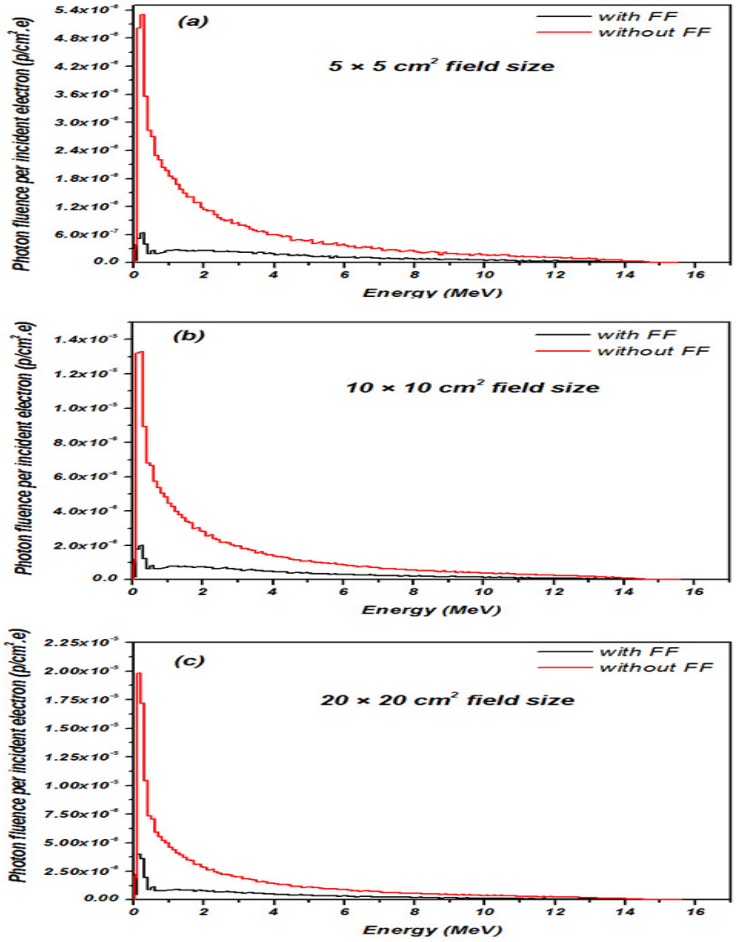

Figure 3: Photon energy spectra for with filter and non-filter case $18 \mathrm{MV}$ photon beam on the phantom surface for different field sizes: (a) $5 \times 5 \mathrm{~cm}^{2}$, (b) $10 \times 10 \mathrm{~cm}^{2}$ and (c) $20 \times 20 \mathrm{~cm}^{2}$. 



Figure 4: Electron energy spectra for with filter and non-filter case $18 \mathrm{MV}$ photon beam on the phantom surface for different field sizes: (a) for $5 \times 5 \mathrm{~cm}^{2}$, (b) for $10 \times 10 \mathrm{~cm}^{2}$ and (c) for $20 \times 20 \mathrm{~cm}^{2}$.

\section{Conclusion}

The photoneutron production in the Siemens Primus Plus medical linac (18 MV photon beam) in a $10 \times 10 \mathrm{~cm}^{2}$ field was calculated and presented. When the field size increased, the photoneutron production is decreased. When the field size increases from $5 \times 5 \mathrm{~cm}^{2}$ to $20 \times 20 \mathrm{~cm}^{2}$, a $17.93 \%$ decrease in the photoneutron production was seen.

The neutron source strength depends on the accelerator model, field size, photon energy, geometry of the simulation and the computational code. The results show that, with increasing field size, the neutron production from jaw was reduced. The reason for this trend is that, by increasing the radiation field, the jaws' opening angle increases and, therefore, less material is located in the path of the photons. For this reason, the number of photon interactions with tungsten inside the jaws decreases and the number of neutrons produced is lower.

The data of neutron source strength in Table 5 are a part of all the published $Q$ values to date. This data will facilitate physicists to accomplish shielding calculations and with this data, physicists can have a better choice in the preparation of accelerators. According to the data in Table 5, neutron production in the Siemens Primus Plus medical linac's head is lower than other accelerators and its usage is recommended.

\section{Acknowledgment}

The authors would like to give thanks to the Hakim Sabzevari University for providing the conditions for using their computer site.

\section{Conflict of Interest}

None

\section{References}

1. Vega-Carrillo HR, Martinez-Ovalle SA, Lallena AM, Mercado GA, Benites-Rengifo JL. Neutron and photon spectra in LINACs. Appl Radiat Isot. 2012;71 Suppl:75-80. doi: 10.1016/j.apradiso.2012.03.034. PubMed PMID: 22494894.

2. Alfuraih A, Chin M, Spyrou N. Measurements of the photonuclear neutron yield of 15 MV medical linear accelerator. Journal of Radioanalytical and Nuclear Chemistry. 2008;278:681-4.

3. Naseri A, Mesbahi A. A review on photoneutrons characteristics in radiation therapy with high-energy photon beams. Rep Pract Oncol Radiother. 2010;15:138-44. doi: 10.1016/j. rpor.2010.08.003. PubMed PMID: 24376940. PubMed PMCID: PMC3863143.

4. Ghiasi H, Mesbahi A. Monte Carlo characterization of photoneutrons in the radiation therapy with high energy photons: a Comparison between simplified and full Monte Carlo models. International Journal of Radiation Research. 2010;8:187.

5. Akkurt I, Adler J0, Annand JR, Fasolo F, 
Hansen K, Isaksson L, et al. Photoneutron yields from tungsten in the energy range of the giant dipole resonance. Phys Med Biol. 2003;48:3345-52. PubMed PMID: 14620062.

6. ICRP. The 2007 Recommendations of the International Commission on Radiological Protection. Oxford: Ann ICRP; 2007. p. 1-332.

7. Becker J. Simulation of neutron production at a medical linear accelerator. Institute of Experimental Physics University of Hamburg, MSc Diploma Thesis. 2007:28-30.

8. Nyandoto P, Muhonen T, Joensuu H. Second cancer among long-term survivors from Hodgkin's disease. Int $J$ Radiat Oncol Biol Phys. 1998;42:373-8. PubMed PMID: 9788418.

9. Kleinerman RA, Boice Jr JD, Storm HH, Sparen P, Andersen A, Pukkala E, et al. Second primary cancer after treatment for cervical cancer. An international cancer registries study. Cancer. 1995;76:442-52. PubMed PMID: 8625126.

10.Chaturvedi AK, Engels EA, Gilbert ES, Chen $B E$, Storm H, Lynch CF, et al. Second cancers among 104,760 survivors of cervical cancer: evaluation of long-term risk. J Natl Cancer Inst. 2007;99:1634-43. doi: 10.1093/jnci/ djm201. PubMed PMID: 17971527.

11.Boice Jr JD, Day N, Andersen A, Brinton L, Brown R, Choi N, et al. Second cancers following radiation treatment for cervical cancer. An international collaboration among cancer registries. J Natl Cancer Inst. 1985;74:95575.

12.Zabihinpoor S, Hasheminia M. Calculation of neutron contamination from medical linear accelerator in treatment room. Adv Studies Theor Phys. 2011;5:421-8.

13.Martinez-Ovalle SA, Barquero R, Gomez-Ros JM, Lallena AM. Ambient neutron dose equivalent outside concrete vault rooms for 15 and 18 MV radiotherapy accelerators. Radiat Prot Dosimetry. 2012;148:457-64. doi: 10.1093/ rpd/ncr208. PubMed PMID: 21750004.

14.Ma A, Awotwi-Pratt J, Alghamdi A, Alfuraih A, Spyrou N. Monte Carlo study of photoneutron production in the Varian Clinac 2100C linac. Journal of Radioanalytical and Nuclear Chemistry. 2007;276:119-23.
15.Mesbahi A. A Monte Carlo study on neutron and electron contamination of an unflattened 18-MV photon beam. Appl Radiat Isot. 2009;67:55-60. doi: 10.1016/j.apradiso.2008.07.013. PubMed PMID: 18760613.

16.Pena J, Franco L, Gomez F, Iglesias A, Pardo J, Pombar M. Monte Carlo study of Siemens PRIMUS photoneutron production. Phys Med Biol. 2005;50:5921-33. doi: 10.1088/00319155/50/24/011. PubMed PMID: 16333164.

17.Dowlatabadi H, Mowlavi AA, Ghorbani M. Monte Carlo Simulation of Siemens Primus plus Linac for 6 and 18 MV Photon Beams. J Biomed Phys Eng. 2017;7:333-46. PubMed PMID: 29616199. PubMed PMCID: PMC5809928.

18.McGinley PH, Landry JC. Neutron contamination of $\mathrm{X}$-ray beams produced by the Varian Clinac 1800. Phys Med Biol. 1989;34:777.

19.Mao XS, Kase KR, Liu JC, Nelson WR, Kleck $\mathrm{JH}$, Johnsen S. Neutron sources in the Varian Clinac 2100C/2300C medical accelerator calculated by the EGS4 code. Health Phys. 1997;72:524-9. PubMed PMID: 9119676.

20. International Atomic Energy Agency. Radiation protection in the design of radiotherapy facilities: Internat. Geneva: Atomic Energy Agency; 2006.

21.Kry SF, Titt U, Ponisch F, Vassiliev ON, Salehpour M, Gillin M, et al. Reduced neutron production through use of a flatteningfilter-free accelerator. Int J Radiat Oncol Biol Phys. 2007;68:1260-4. doi: 10.1016/j. ijrobp.2007.04.002. PubMed PMID: 17637397.

22.Lin JP, Chu TC, Lin SY, Liu MT. The measurement of photoneutrons in the vicinity of a Siemens Primus linear accelerator. Appl Radiat Isot. 2001;55(3):315-21. PubMed PMID: 11515653.

23.Becker J, Brunckhorst E, Schmidt R. Photoneutron production of a Siemens Primus linear accelerator studied by Monte Carlo methods and a paired magnesium and boron coated magnesium ionization chamber system. Phys Med Biol. 2007;52(21):6375-87. PubMed PMID: 17951849.

24.Followill DS, Stovall MS, Kry SF, Ibbott GS. Neutron source strength measurements for 
Dowlatabadi H. et al

Varian, Siemens, Elekta, and General Electric linear accelerators. J Appl Clin Med Phys. 2003;4(3):189-94. PubMed PMID: 12841788. PubMed PMCID: PMC5724447.

25.Mohammadi N, Miri-Hakimabad H, RafatMotavlli L, et al. Neutron spectrometry and determination of neutron contamination around the 15 MV Siemens Primus LINAC. $J$ Radioanal Nucl Chem. 2015;304:1001-8. doi: 10.1007/s10967-015-3944-5.

26.McCall RC. Neutron yields of medical electron accelerators. Report SLAC-PUB 4480; United States: Stanford Linear Accelerator Center; 1987. 